\title{
Selección de Estrategias de Mejoramiento de las Condiciones de Trabajo para la Función Mantenimiento Utilizando la Metodología MCDA Constructivista
}

\author{
Fernando F. Espinosa y Gonzalo E. Salinas \\ Facultad de Ingeniería, Universidad de Talca, 2 Norte 685, Talca-Chile (e-mail: fespinos@utalca.cl; \\ gsalinas@utalca.cl)
}

Recibido Nov. 20, 2012; Aceptado Ene. 09, 2013; Versión final recibida Ene. 16, 2013

\begin{abstract}
Resumen
Se presenta una herramienta de análisis cuya finalidad es definir que estrategias son las más adecuadas para ser implementadas en una organización productiva a fin de producir un cambio efectivo en las condiciones de trabajo para las personas que están a cargo de la función mantenimiento. Para lograr tal efecto se combinaron herramientas de la Metodología Multicriterio de Apoyo a la Decisión-Constructivista (MCDA) para organizar y sintetizar la información recopilada desde el equipo de personas, del modelo DEMATEL (Decision Making Trial and Evaluation Laboratory) para jerarquizar las estrategias definidas y finalmente del análisis de regresión como herramienta de seguimiento de la evolución de las acciones que serán parte de las estrategias. Lo más relevante de la aplicación de este procedimiento es que la definición de las estrategias nace desde las personas que laboran en el área de mantenimiento lo que conlleva a que el compromiso con la implementación exitosa de estas acciones sea muy alto.
\end{abstract}

\section{Selection of Improvement Strategies for Working Condition for to Maintenance Function Using Constructivist Multicriteria Decision Aid Approach MCDA}

\begin{abstract}
It's presented an analysis tool in order to define which strategies are most suitable for to be implemented, which would lead to an effective change in the actual working conditions for the people who are in charge of the maintenance function in a productive organization. To this purpose tools of the Constructivist Multicriteria Decision Aid Approach (MCDA) to organize and synthesize the information gathered from the maintenance working team, the DEMATEL model (Decision Making Trial and Evaluation Laboratory) to prioritize the defined strategies and the regression analysis as a tool for monitoring the evolution of the actions that will be part of the strategies. The most important aspect of this procedure is that the definition of the strategies comes from the same people who work in the maintenance area, fact which makes that the commitment with the successful implementation of such strategies be very high.
\end{abstract}




\section{INTRODUCCION}

Entre las tareas que competen a un administrador del mantenimiento está la de proponer estrategias que ayuden a conseguir un mejoramiento cualitativo del servicio que entrega para su empresa. Este mejoramiento, que debe tener impacto directo y positivo en su gestión, tiene múltiples facetas producto de los variados requerimientos que están presentes como variables en su proceso de toma de decisiones, como ser cantidad y calidad de recursos físicos y humanos, tipo de información a usar, apoyo del entorno, condiciones laborales, etc. (Espinosa y Salinas, 2010).

Para decidir cuales deberían ser las tareas a realizar, con la finalidad de optimizar la gestión de sus recursos, se puede proponer un modelo y simular una programación multicriterio (; Xu y Ouenniche, 2012; Okeola y Sule, 2011; Martinez et al., 2006; Mavrotas y Trifillis, 2006; Mavrotas et al., 2006; Løken, 2005). Cuando se considera ese tipo de problemas, claramente se puede apreciar que los métodos exactos quedan limitados en su tamaño a causa de los requerimientos de recursos y tiempo (Roux et al., 2007; Hites et al., 2005), por tanto el método seleccionado debe ser capaz de describir con bastante exactitud los problemas reales y llevar hacia una solución, en un tiempo y costo razonable.

Aprovechar el conocimiento y juicio del administrador y su equipo de personas, con bastante seguridad, proveerán información muy útil para la toma de decisiones en lo que respecta a la definición de las estrategias de mejoramiento más acertadas para la realidad de la gestión. Cuando se procede de esta manera se pueden combinar múltiples visiones del problema y así tener varios puntos de referencias sobre los cuales se pueden aplicar variados métodos de ajustes (Winkler y Clemen, 2004). Cuando se desea capturar la visión del experto, el analista debe definir cual es el mejor método para obtener dicha información.

Las herramientas del MCDA Constructivista ayudan a organizar y sintetizar la información que ha sido recopilada desde los involucrados directamente con el problema en estudio (Hodgkin et al., 2005) y además se puede de forma natural combinarse con un modelo de administración que maneje la incerteza (Løken, 2007; Linkov et al., 2006). Este hecho permite obtener un conjunto de alternativas que podrían ser tratadas de forma dinámica y así obtener información sobre los efectos de los diferentes cursos de acción y apoyar la toma de decisiones, ya que el equipo de análisis puede organizar la búsqueda de las soluciones que optimicen la probabilidad de éxito, el tiempo y el costo con los cuales se consigue una solución que aporta valor a la organización (Goncalves et al., 2012; Ensslin et al., 2008; Nickerson y Zenger, 2004).

En el área del mantenimiento el modelo MCDA Constructivista tiene aplicaciones para generar auditorias para mantenimiento preventivo (Bana e Costa, 2012), generación de prioridades para las actividades de reparación (Bana e Costa, 2002) y evaluación del mantenimiento de infraestructuras (Gervásio y, Simões da Silva, 2012). En este trabajo se aplicará parte del procedimiento de la metodología MCDA, para definir las áreas de preocupación de las personas que ejecutan los programas de mantenimiento en sus respectivas empresas, para las condiciones actuales de trabajo y las acciones que son necesarias implementar para introducir mejoramientos en los múltiples factores que influyen en la eficiencia del mantenimiento,

Para complementar el proceso de selección de las estrategias y acciones de mejoramiento se usarán los principios, del modelo DEMATEL para jerarquizar las estrategias (Wang et al,. 2012; Hu et al., 2011; Lin et al., 2011) y de esta manera priorizar los recursos financieros disponibles para invertir en el mejoramiento de las condiciones del entorno de la función mantenimiento. Se complementa el modelo con el análisis de regresión como herramienta de seguimiento de la evolución de las acciones (Lee y Akin, 2009; Kwon, 2001;) las que serán implementadas por los usuarios para el mejoramiento de la gestión del mantenimiento y así tener puntos para el control de estas y análisis de los desvíos.

\section{PROCEDIMIENTO PARA DEFINIR LOS PUNTOS DE VISTA FUNDAMENTALES}

La metodología MCDA tiene como objetivo auxiliar en la toma de decisión, para un determinado problema, según la perspectiva de los decisores (responsables por la solución del problema, o bien, los principales afectados por el problema en cuestión). Según Ensslin et al. (2001) los procedimientos deben estar fundamentados en un paradigma que defina cuales son las reglas de trabajo para garantizar el respaldo científico a la metodología.

El procedimiento que se usó en esta aplicación es descrito en la Fig. 1 y está compuesto de tres grandes grupos de actividades: identificación del contexto decisorio, estructuración del problema y finalmente la definición de las estrategias para el mejoramiento. En la fase de estructuración, es donde se aplica más en profundidad la metodología MCDA y es aquí donde es recolectada la información para entender mejor el 
problema, de forma de estructurar ese conocimiento para que éste represente las perspectivas de los involucrados con el problema, o sea, los decisores. El primer paso es caracterizar el contexto de la decisión, definiendo el contorno del problema, o sea, sus relaciones internas y límites y las personas afectadas. La definición del contexto decisorio va a identificar a los actores involucrados directa y/o indirectamente en el proceso y esos actores son personas o grupos que entregarán las acciones, valores o alternativas en la construcción del modelo multicriterio.

En base al contexto decisorio se define un rótulo para el problema y este tiene como objetivo orientar la identificación de los elementos primarios de evaluación (EPEs), delimitando este contexto. Los EPEs representan objetivos, metas, valores y opciones de los decisores los cuales son utilizados como base para la construcción de los mapas de medio - fin. A partir de los EPEs son construidos los conceptos para llevarlos a la acción, que es la parte central de la segunda fase de la metodología.

La obtención de los EPEs se realizó por medio de entrevistas a los encargados del mantenimiento de tres empresas productivas que representan el potencial productivo de la zona, una del área de la celulosa y dos de la producción del vino, las que fueron apoyadas por una guía que contenía aquellos aspectos que en general son los más sensibles para quienes realizan el trabajo cotidiano del mantenimiento, y de esta forma facilitar la tarea de los decisores y organizar el trabajo del facilitador tratando de abarcar lo más posible el amplio espectro de posibilidades que el tema entrega. El rótulo y los EPEs indicados por los mantenedores se resumen en la Fig. 2.

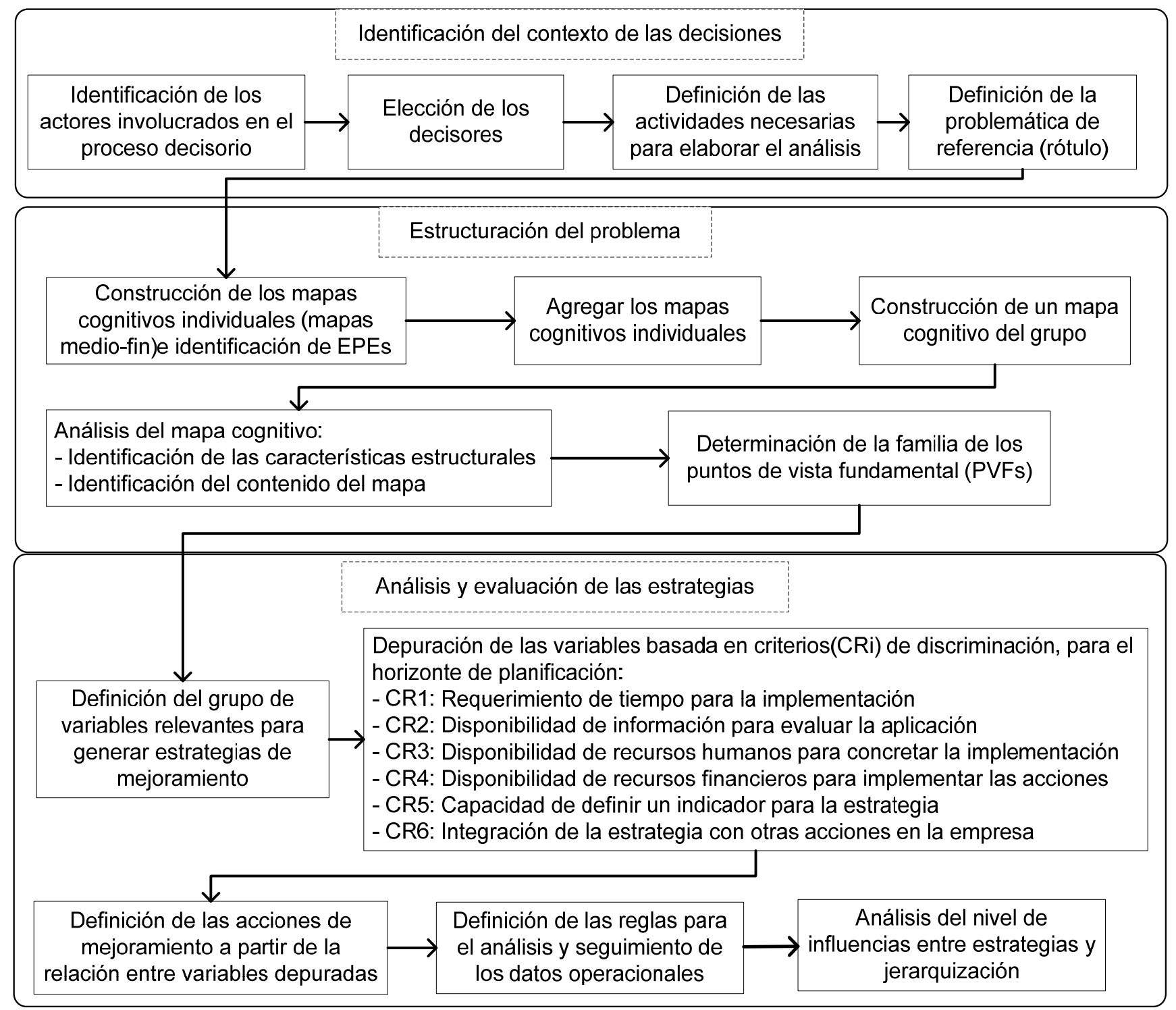

Fig. 1: Procedimiento para definir las estrategias de mejoramiento. 
En los modelos constructivistas, la representación del proceso mental del problema, según el decisor, es hecha por medio de los mapas medio - fin. Estos son construidos en base en los conceptos medios, conceptos fin y relaciones de influencia. A partir de un concepto dado, el facilitador cuestiona al decisor utilizando el adverbio - cómo - (por ej.: ¿cómo se puede obtener tal concepto?), indicando que tal concepto va direccionado al medio. Cuando el facilitador utiliza la conjunción adverbial - por qué - (por ej.: ¿por qué ese concepto es importante?), indica que el concepto está encaminado en dirección al fin. La relación de influencia es representada por flechas que unen los conceptos entre sí, donde, se parte de un concepto medio hacia un concepto fin (Fig. 3). El desarrollo del procedimiento propuesto será ejemplificado para este trabajo, utilizando los conceptos definidos para el aspecto financiero.

(1) Tener mayor disponibilidad de tiempo para el control del mantenimiento

(2) Agregar calidad al producto

(3) Tener las capacidades para mejorar los equipos

(4) Tener planes de mantenimiento anuales completos
(37) Tener tiempo para la evaluación de los gastos diarios

(36) Contar con información financiera actualizada para tomar una buena decisión

(35) Tener mayor apoyo para la función mantenimiento por parte de gerencia

(34) Tener control de los gastos en mantenimiento por máquina

(33) Tener inversión en nuevas tecnologías

(5) Tener más control sobre la ejecución de los trabajos

(6) Tener mayor supervisión de los trabajos

(7) Tener mayor cantidad de personal

(8) Tener mayor apoyo de la jefatura de operaciones

(9) Tener control sobre el presupuesto asignado

(10) Conseguir mayor cooperación de la gente de producción

(11) Tener mayor orden en el área de mantenimiento

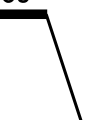

(12) Tener cooperación de ingenieria
antes y después en un proyecto
(13) Realizar los mantenimientos
en el costo estipulado

(14) Contar con información actualizada de los equipos

(15) Determinar la capacidad de nuevos recursos humanos

(16) Tener control sobre las actividades de terceros

(17) Tener definida la criticidad de los equipos con mayor rigurosidad

(18) No sobrepasarse del presupuesto fijado para el periodo

Evaluar el desempeño de la
(32) Tener personal con mayor calificación

(31) Responder a las contingencias con rapidez

(30) Tener incentivos para los operadores que cooperen con mantenimiento

(29) Tener más alternativas de servicios de terceros

(28) Tener disponibilidad de gestión de mantenimiento actual de la empresa procurando identificar nuevos abordajes estratégicos. tiempo para capacitar al personal

(27) Tener mayor cantidad de recursos humanos

(26) Tener antecedentes validados para elaborar presupuestos

(25) Tener los medios para calificar los trabajos realizados por terceros

(24) Respetar el calendario planificados para las paradas de planta

(23) Utilizar software para el control de la ejecución de las tareas

(22) Inversión en repuestos que dan resultado

(21) Tener buen tiempo de reacción en la gestión de repuestos

(20) Tener apoyo continuo de gerencia

(19) Tener mayor certeza sobre los gastos que se van a incurrir

Fig. 2: Rótulo del problema y elementos primarios de evaluación 
Con base en los mapas de medio-fin son identificados los conceptos interrelacionados que explican una preocupación común los cuales son agrupados en clúster. Los clúster están compuestos por secuencias de conceptos unidos por relaciones de influencia que tratan de asuntos similares, las ramas, a partir de las cuales se inicia el proceso de transición y cada rama va a generar un eje de evaluación del problema. En la Fig. 4 se muestra un sub-conjunto de un clúster obtenido para los elementos primarios agrupados del aspecto financiero, el cual será tratado en extenso en este trabajo, y en la Fig. 5 está la totalidad de los clúster identificados en esta aplicación.

Basado en las ramas contenidas en los mapas, son definidos los puntos de vista fundamentales (PVFs) los cuales representan las perspectivas de los decisores para el problema en cuestión. Estos PVFs según Ensslin et al. (2001), son aspectos considerados como fundamentales por los decisores, para evaluar las acciones potenciales, ya que ellos explican los valores que los decisores consideran importantes en aquel contexto y al mismo tiempo definen las características de las acciones que son de interés de los decisores.

\section{DEFINICIÓN DE ESTRATEGIAS EN BASE A LOS MAPAS MEDIO-FIN.}

El siguiente paso en el desarrollo de la metodología propuesta es la definición de las estrategias que conducirán al mejoramiento de aquellos aspectos, que según los implicados, son trabas para una buena gestión del mantenimiento. La definición e implementación correcta de las estrategias de forma exitosa es vital para cualquier organización, pero transformar estrategias en acción es una tarea compleja y difícil (Aaltonen y lkavalko, 2002), y a lo anterior se suma el hecho que debido a la creciente dependencia de la tecnología y los requerimientos de los usuarios, el replanteamiento de estrategias y la medición de su efectividad es una necesidad permanente para la función de mantenimiento (Singh et al. 2002; Tsang, 2002; Madu, 2000).

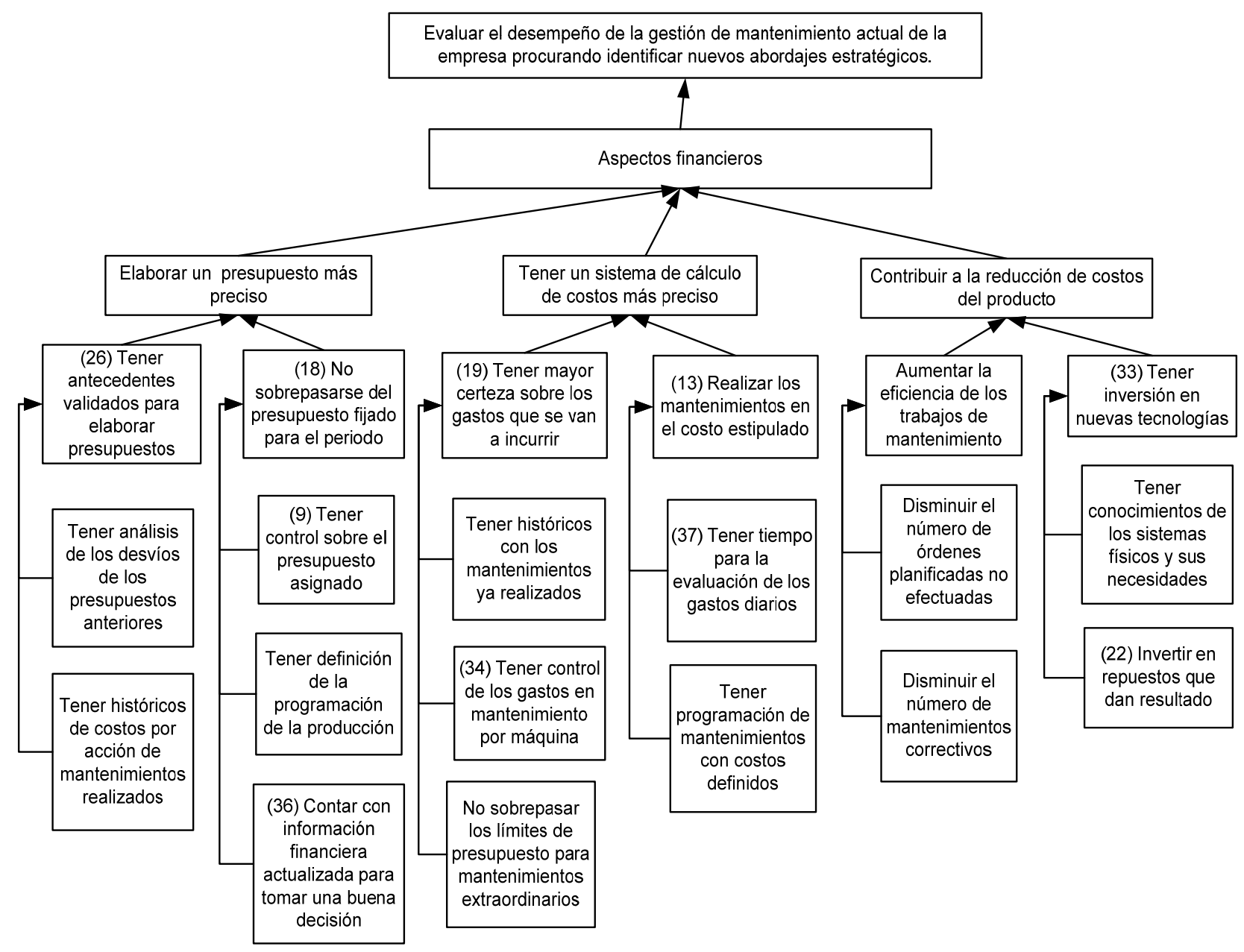

Fig. 3: Mapa medio - fin para el aspecto financiero 
El desarrollo del procedimiento llega hasta el punto donde los involucrados proponen las estrategias y las validan como factibles de ser implementadas, pero, la implementación es un proceso que toma tiempo para ver sus resultados, sólo que ahora la definición nace de un análisis de las causas que ellos mismos evalúan como importantes o un problema evidente (Büyükdamgaci, 2003). Cuando se analizan las distintas variables del proceso de una manera sinérgica se puede seguir en el proceso de búsqueda de aquellas alternativas que mejor responden a los requerimientos de los usuarios del sistema (Okumus, 2001).

En el proceso de obtención de los elementos primarios que emergen de las distintas entrevistas y validaciones por parte de los decisores al jerarquizarlos, en los mapas medio - fin, se da el hecho que el nivel superior es función de los conceptos directamente relacionados en el nivel inferior inmediato, como se puede observar en la Fig. 4. La definición de las estrategias se inicia con la pregunta de cómo llevar a acciones los conceptos designados como variables independientes y correlacionarlos con el concepto superior el cual es directamente dependiente de estas acciones, y es aquí donde el grado de creatividad de los involucrados en el problema decide cual información es transformada en conocimiento, sobre lo cual la acción será definida (Johannessen et al., 1999)

Realizar un proceso de mejoramiento continuo, para lo cual este procedimiento está dirigido como herramienta de apoyo, debe formar parte de la cultura organizacional que apunta a eliminar las debilidades en todo el sistema (Bhuiyan y Baghel, 2005; Andriani y Hall, 2003) donde se debe aprovechar todo el capital humano que la empresa posee para transformar el conocimiento tácito adquirido mediante la experiencia en conocimiento explicito codificado que facilitará la comunicación entre todos los estamentos de la organización

Revisando todos los elementos primarios que son finalmente definidos después de varias iteraciones, en especial aquellos denominados como variables independientes, mediante un proceso similar al usado para definir estos elementos se listan aquellas acciones que podrían ser implementadas para atenderlos, teniendo presente que el objetivo es conformar un conjunto de estrategias destinadas al mejoramiento de las condiciones de trabajo de la función mantenimiento, por tanto deben ser acciones que tengan la factibilidad de ser implementadas, tomando como referencia los criterios (CRi) definidos en la Fig. 1.

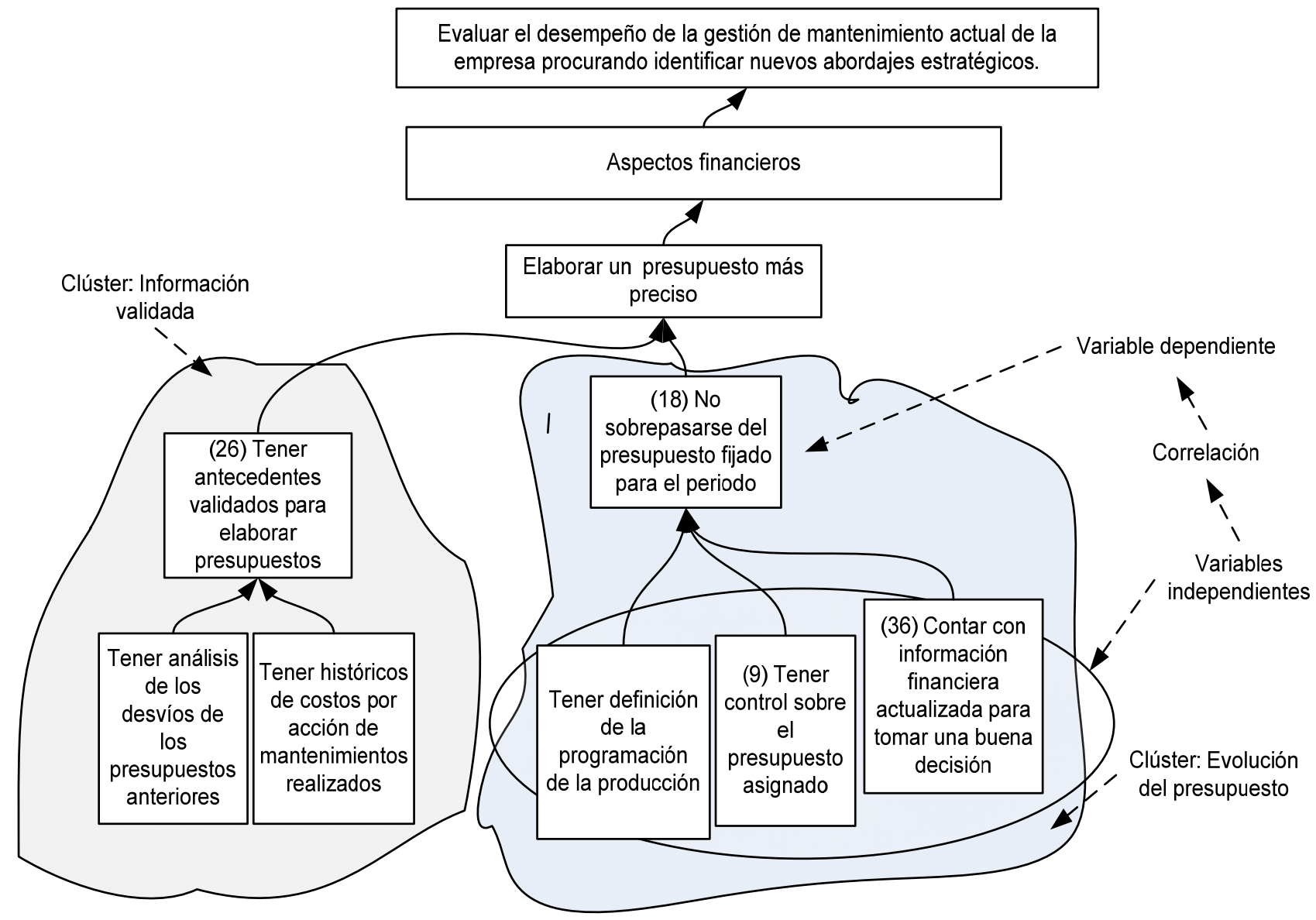

Fig. 4: Ejemplo de variables dependientes e independientes para cada clúster 
Como generalmente se da que existe más de una estrategia para conseguir el objetivo asociado a la variable dependiente, es necesaria la depuración de las acciones con posibilidad de ser implementadas, a fin de concentrar los esfuerzos en aquellas que en su conjunto darán un resultado esperado mayor. Para tal fin se hace necesario jerarquizar estas acciones mediante un análisis estructurado y participativo tal que el integrante de la organización conozca lo que es importante para el desarrollo de su lugar de trabajo (Kutucuoglu et al., 2001), por lo cual, es fundamental que las decisiones sobre las prioridades de estas estrategias se basen en criterios razonables y objetivos que reflejen las políticas definidas y perseguidas por el grupo de mantenimiento (Bana e Costa y Carvalho, 2002).

A continuación, en la tabla 1, se presenta el listado de variables obtenidas de los mapas medio - fin para cada área de los aspectos financieros, y para la depuración de ellas se usó la siguiente la valoración: +1, si es aplicable con facilidad; 0 , si es aplicable con condicionamientos especiales y - 1 , si no es aplicable, la cual está definida por las condiciones actuales de disponibilidad de recursos, tiempo, conocimientos o requerimientos específicos. En caso que dos de las variables independientes tuvieran una misma ponderación y fueran las mejor calificadas, la elección depende directamente del decisor en base a la aplicabilidad y capacidad de emprender las acciones que esta selección conlleva.

\section{AJUSTE DE LAS ESTRATEGIAS DE MEJORAMIENTO}

El proceso de implementación de mejoras de las condiciones para la función mantenimiento se puede dividir en dos partes: la definición de las estrategias y la implementación de estas. La primera parte, la definición, que es el producto final de esta metodología, requiere del conocimiento del efecto que las acciones a implementar tendrán sobre los objetivos del mantenimiento, como un insumo, los cuales se derivan directamente del plan de negocios. Esta parte inicial condiciona el proceso de gestión para el éxito del mantenimiento en una organización, y determina la eficacia de la posterior aplicación de los planes, controles y mejoras (Crespo et al., 2009).

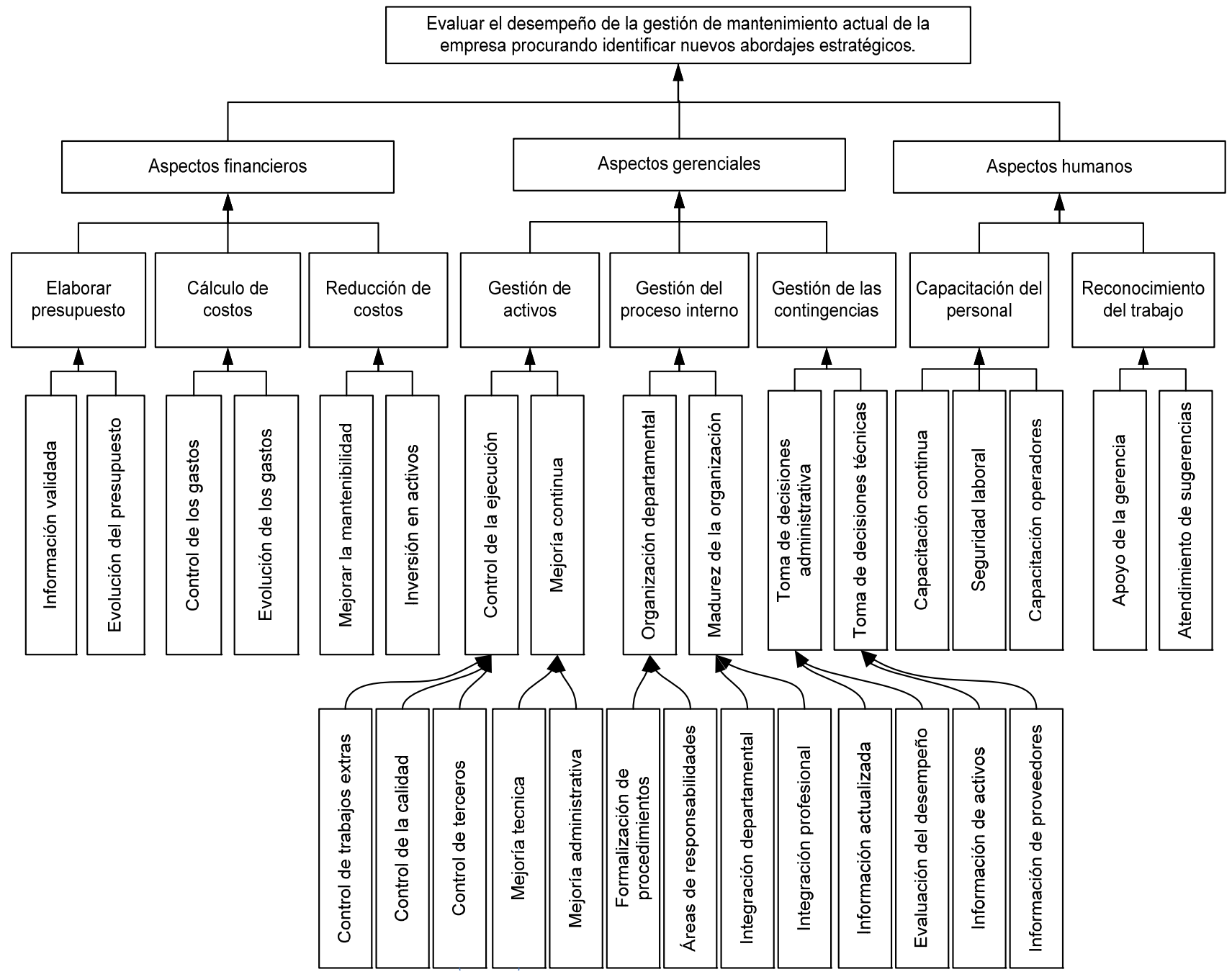

Fig. 5: Totalidad de los clúster definidos para el problema analizado. 
La segunda parte del proceso, la aplicación de la estrategia seleccionada tiene un nivel de importancia diferente. Se refiere a la capacidad de la organización para hacer frente al problema de la gestión de la aplicación de las acciones de mejoramiento (por ejemplo, la capacidad de asegurar los niveles adecuados de habilidades, de preparación del trabajo, de herramientas y del cumplimiento del programa), que va a permitir reducir al mínimo las falencias o debilidades de la función mantenimiento.

Una estrategia se compone de una serie de acciones, consolidadas en un plan maestro para su coherencia e integridad, y de esa forma asegurar y demostrar su contribución a los objetivos estratégicos corporativos y de negocios (Nollet et al, 2005). Así, los resultados de la aplicación una estrategia se debería manifestar como la reducción de la varianza, mejora de los ciclos de tiempo, minimización del costo total, uso de las tecnología de la información para captura de datos y monitoreo y un programa administrado para la actividad.

Tabla 1: Listado de variables dependientes e independientes para el aspecto financiero

\begin{tabular}{|c|c|c|c|c|c|c|c|}
\hline Elaborar un presupuesto más preciso & CR1 & CR2 & CR3 & CR4 & CR5 & CR6 & SUMA \\
\hline $\begin{array}{l}\text { Tener antecedentes validados para elaborar } \\
\text { presupuestos (dep.) }\end{array}$ & -- & -- & -- & -- & -- & -- & -- \\
\hline $\begin{array}{l}\text { a. Tener análisis de los desvíos de los presupuestos } \\
\text { anteriores (indep.) }\end{array}$ & +1 & -1 & +1 & +1 & 0 & +1 & +3 \\
\hline $\begin{array}{l}\text { b. Tener históricos de costos por acción de } \\
\text { mantenimientos realizados (indep.) }\end{array}$ & +1 & +1 & +1 & +1 & +1 & 0 & +5 \\
\hline $\begin{array}{l}\text { No sobrepasarse del presupuesto fijado para el periodo } \\
\text { (dep.) }\end{array}$ & -- & -- & -- & -- & -- & -- & -- \\
\hline a. Tener control sobre el presupuesto asignado (indep.) & 0 & -1 & +1 & +1 & +1 & +1 & +3 \\
\hline $\begin{array}{l}\text { b. Tener definición de la programación de la producción } \\
\text { (indep.) }\end{array}$ & +1 & -1 & -1 & +1 & -1 & +1 & 0 \\
\hline $\begin{array}{l}\text { c. Contar con información financiera actualizada para } \\
\text { tomar una buena decisión (indep.) }\end{array}$ & 0 & -1 & +1 & +1 & +1 & +1 & +3 \\
\hline Tener un sistema de cálculo de costos más preciso & CR1 & CR2 & CR3 & CR4 & CR5 & CR6 & SUMA \\
\hline $\begin{array}{l}\text { Tener mayor certeza sobre los gastos que se van a } \\
\text { incurrir (dep.) }\end{array}$ & -- & -- & -- & -- & -- & -- & -- \\
\hline $\begin{array}{l}\text { a. Tener históricos con los mantenimientos ya } \\
\text { realizados (indep.) }\end{array}$ & +1 & +1 & +1 & 0 & +1 & +1 & +5 \\
\hline $\begin{array}{l}\text { b. Tener control de los gastos en mantenimiento por } \\
\text { máquina (indep.) }\end{array}$ & +1 & -1 & -1 & +1 & -1 & +1 & 0 \\
\hline $\begin{array}{l}\text { c. No sobrepasar los límites de presupuesto para } \\
\text { mantenimientos extraordinarios (indep.) }\end{array}$ & +1 & -1 & -1 & +1 & -1 & +1 & 0 \\
\hline Realizar los mantenimientos en el costo estipulado (dep.) & -- & -- & -- & -- & -- & -- & -- \\
\hline $\begin{array}{l}\text { a. Tener tiempo para la evaluación de los gastos diarios } \\
\text { (indep.) }\end{array}$ & 0 & 0 & -1 & 0 & +1 & 0 & 0 \\
\hline $\begin{array}{l}\text { b. Tener programación de mantenimientos con costos } \\
\text { definidos (indep.) }\end{array}$ & +1 & -1 & 0 & +1 & +1 & +1 & +3 \\
\hline Contribuir a la reducción de costos del producto & CR1 & CR2 & CR3 & CR4 & CR5 & CR6 & SUMA \\
\hline $\begin{array}{l}\text { Aumentar la eficiencia de los trabajos de mantenimiento } \\
\text { (dep.) }\end{array}$ & -- & -- & -- & -- & -- & -- & -- \\
\hline $\begin{array}{l}\text { a. Disminuir el número de órdenes planificadas no } \\
\text { efectuadas (indep.) }\end{array}$ & +1 & +1 & 0 & 0 & +1 & +1 & +4 \\
\hline $\begin{array}{l}\text { b. Disminuir el número de mantenimientos correctivos } \\
\text { (indep.) }\end{array}$ & +1 & -1 & -1 & -1 & +1 & 0 & -1 \\
\hline $\begin{array}{l}\text { Tener inversión de recursos en nuevas tecnologías } \\
\text { (dep.) }\end{array}$ & -- & -- & -- & -- & -- & -- & -- \\
\hline $\begin{array}{l}\text { a. Tener conocimientos de los sistemas físicos y sus } \\
\text { necesidades (indep.) }\end{array}$ & -1 & -1 & +1 & 0 & +1 & 0 & 0 \\
\hline $\begin{array}{l}\text { b. Invertir recursos monetarios en repuestos que dan } \\
\text { resultado (indep.) }\end{array}$ & 0 & 0 & +1 & +1 & +1 & 0 & +3 \\
\hline
\end{tabular}

La estrategia es lo que transforma las ideas en realidad. Sin ideas no hay un contexto para la formulación e implementación de estrategias (Chaharbaghi y Willis, 1998). Para la función mantenimiento las ideas para 
formular las estrategias vienen del conocimiento en terreno de la realidad que se enfrenta el personal y que debe mantener bajo su control. Además de eso, se suma el hecho de que la implementación de una estrategia es un proceso que cubre un período de tiempo y, por tanto necesita de un aprendizaje y de un seguimiento continuo. Es por eso que el manejo de correlaciones entre el factor clave de éxito definido y la tasa de variación del efecto que se desea optimizar, entrega un mejor diagnóstico de la efectividad de las acciones implementadas para la mejora del mantenimiento.

El conocimiento es parte fundamental en todo tipo de organizaciones, tanto de manufactura como de servicios, pero es especialmente relevante en organizaciones que producen o que necesitan de una gran cantidad de conocimiento especializado para realizar sus operaciones (Uriona y Varvakis, 2008). En las organizaciones dedicadas al mantenimiento, el conocimiento no solo debe ser referido a la aplicación de estos conocimientos sobre los activos ligados a los procesos productivos sino que también de los procesos administrativos internos, y que incluyen el conocimiento explícito y tácito distribuidos entre sus integrantes los cuales deberían formar la estructura básica en la explicitación de las estrategias de mejoramiento.

La probabilidad de obtener mejores soluciones aumenta cuando la investigación avanza a partir de puntos que son conocidos y sus resultados se entienden mejor. Esta es la razón para adquirir conocimientos y discutir los problemas con los demás integrantes del equipo de trabajo (Huang, 2011). Independientemente de los tipos de conocimiento, siempre existen posibilidades de aportar ideas brillantes para llegar a las soluciones. A fin de reunir los conocimientos necesarios, es fundamental que los integrantes interactúen organizados por un facilitador ya que los operadores - mantenedores están restringidos por limitaciones de tiempo y espacio, para encontrar la combinación óptima de un número aparentemente ilimitado de fuentes, pero, bajo esta forma de trabajo es posible saber si la combinación es adecuada para los problemas o no.

Una vez identificados los factores que van ser optimizados (minimizar o maximizar), se procura proponer las estrategias que van a ser implementadas para cada uno de los factores claves de éxito. El proceso comienza con la declaración del objetivo que se desea conseguir y para este objetivo se define cual será la estrategia a implementar. En un período posterior se correlacionarán los resultados obtenidos en el ambiente de fábrica, como consecuencia de la ejecución de las acciones y la tasa de variación para un periodo de referencia. Dependiendo de la naturaleza de la tasa y de la estrategia definida, la correlación será positiva o negativa (Sanders y Smidt, 2000), evidentemente, en función del juzgamiento del administrador sobre los resultados y sobre el éxito de la estrategia.

Así, por ejemplo, en la definición de una estrategia para elaborar un presupuesto más preciso, se declara y se verbaliza ésta, en conjunto entre la definición del área de preocupación y la variable dependiente para focalizar el objetivo de la estrategia, que en este caso sería: "Elaborar un presupuesto más preciso vía poseer una información más exacta y actualizada". Las acciones de mejoramiento se declaran o definen a partir de la variable dependiente más la variable independiente elegida que se obtuvieron en las etapas anteriores de esta metodología. Estas acciones tienen que ser definidas de tal manera que puedan ser medibles (o sea controlables) e implementadas dentro de los tiempos de planificación y además del factor de correlación (FC) entre estas variables con la finalidad de actuar de forma positiva sobre este factor. En la Fig. 6 se muestran los aspectos para esta estrategia (E1) y las restantes definidas para el aspecto financiero del problema.

Si el administrador prefiere vincular su gestión a un conjunto de indicadores, el modelo también sirve ya que parte del factor de correlación se puede transformar en un indicador. En ese caso debería tener un valor de referencia para medir la eficiencia de la implementación de la estrategia.

No basta un buen modelo de análisis y el uso de herramientas de efectividad comprobada, sino que también el analista debe tener la capacidad de discriminar e interpretar adecuadamente los resultados para tomar las decisiones acertadas. Además de eso, no puede perder la visión del negocio, o una visión holística de la empresa, tanto en su ámbito interno, como en relación a otras empresas del ramo.

\section{JERARQUIZACIÓN DE LAS ESTRATEGIAS DE MEJORAMIENTO}

El éxito de un programa de mejoramiento radica en la capacidad de la dirección de la función mantenimiento para seleccionar la combinación adecuada de proyectos que maximizan el impacto en el servicio del cual son responsables con menos recursos de los que les fueron asignados. Además, el proceso de identificación de la cartera de proyectos que pueden alcanzar con éxito los múltiples objetivos en condiciones de recursos limitados, es también otra decisión crítica para cualquier organización. 


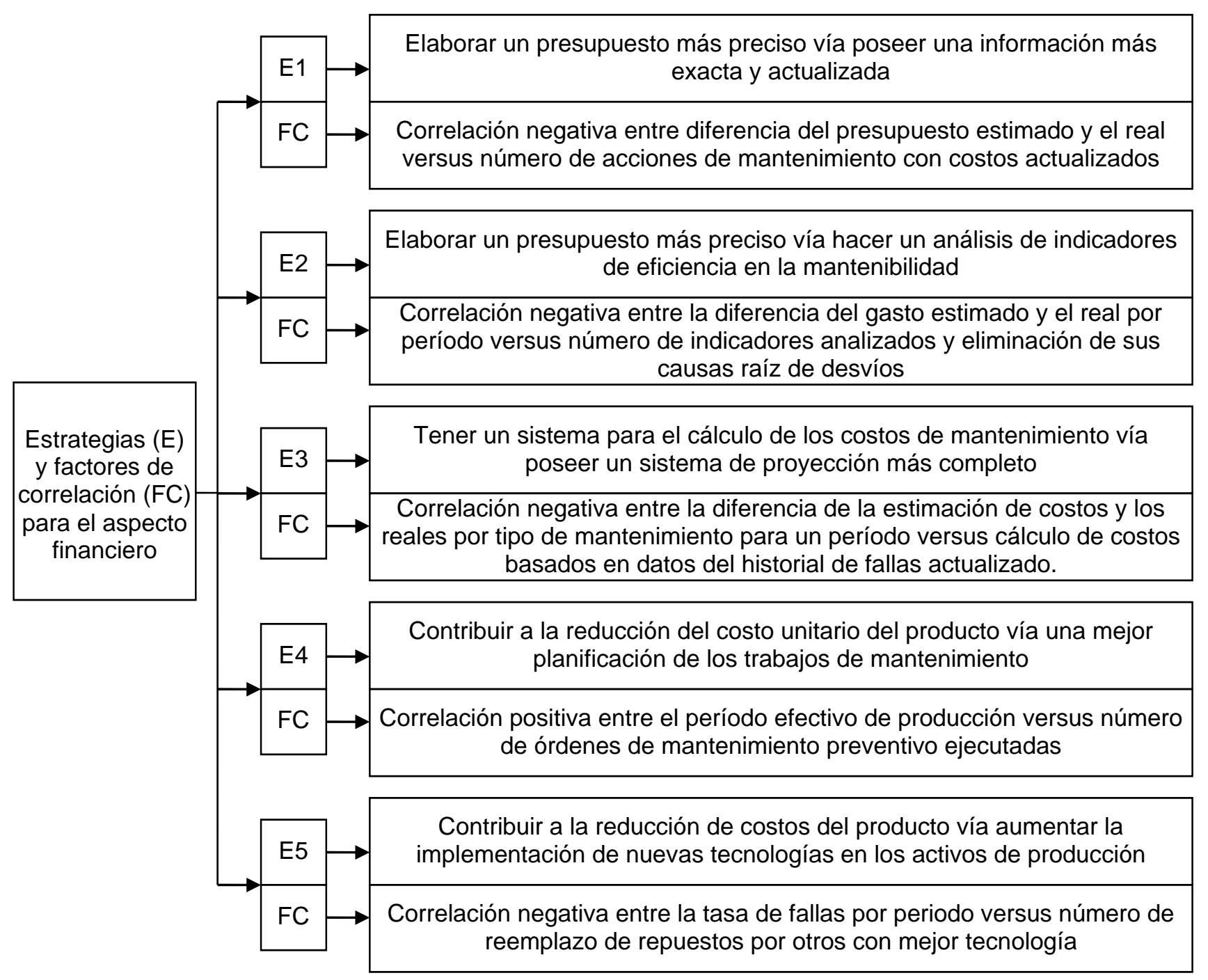

Fig. 6: Datos para el análisis de una estrategia implementada para el aspecto financiero

En el nivel macro los proyectos de mejoramiento pueden ser vistos como proyectos de inversión de capital. Las inversiones de capital comparten tres características comunes importantes: (a) que puede ser parcial o totalmente irreversibles, (b) existe incertidumbre sobre los beneficios futuros de la inversión, y (c) los gerentes tienen cierta libertad de acción sobre el tiempo de la inversión (Padhy y Sahu, 2011). Los beneficios esperados de los proyectos de mejoramiento pueden fluctuar debido a los riesgos relacionados tanto con el proyecto como del entorno. Entonces, como las inversiones en proyectos típicos de mejoramiento podrían estar expuestas a más de una fuente de riesgos, es necesario encontrar la manera de modelar y evaluar dichos proyectos frente a los riesgos asociados (Espinosa et al., 2012). También es muy imprescindible impartir flexibilidad en las decisiones de gestión, mediante la identificación de las distintas opciones incluidas en el proyecto.

Este tipo de aplicación, implica tomar decisiones que se inician en la definición de los objetivos a conseguir, la recopilación de los criterios pertinentes y las posibles alternativas, evaluar las alternativas con sus ventajas y desventajas, y la selección de la alternativa óptima $(\mathrm{Wu}, 2008)$. Hasta este punto de la metodología, en la primera parte se definió la meta que en este problema es seleccionar una estrategia de mejoramiento favorable. En la etapa 2, está el proceso para determinar y establecer los clúster para evaluación. Con el fin de evaluar y seleccionar una estrategia de mejoramiento favorable, los clúster para la evaluación necesarios a ser considerados nacen del análisis y alcance que los propios involucrados en la función mantenimiento consigan definir, pre - seleccionar o bien deseen abarcar.

En la etapa 3, es necesario aplicar un modelo que permita jerarquizar las estrategias de tal forma que el esfuerzo que implica a la organización un proyecto de este tipo sea bien dirigido con una selección final acertada entre todas las alternativas posibles. El modelo que se aplicó fue el modelo DEMATEL que es una metodología para hacer frente a problemas complejos, estructurándolos a través de representaciones 
gráficas y analizando las influencias causales puede ayudar en cuestiones complejas, sistemas o conceptos confirmando la interdependencia entre las variables y de ayuda en el desarrollo de un gráfico para reflejar las interrelaciones entre las variables. El producto final del proceso de DEMATEL es una representación visual del mapa relaciones de influencia (Fig. 7) mediante el cual los administradores organizan sus propias acciones (Büyüközkan y Öztürkcan, 2010). Por último, en el paso 4, las mejores soluciones se pueden ordenar de acuerdo a las prioridades generales de las alternativas.

Los pasos en la aplicación de DEMATEL son como sigue:

Paso 1: Generación de la matriz de relaciones directas (Tabla 2). Evaluación de la relación entre las estrategias y sus criterios basado en una escala de comparación diseñada en cuatro niveles: sin influencia (0), la influencia es baja (1), la influencia es mediana (2), la influencia es alta (3), la influencia es muy alta (4). Al equipo de expertos se le pide hacer las comparaciones por pares en términos de influencia y la dirección entre los criterios. Los resultados de estas evaluaciones forman una matriz $n \times n$ llamada matriz de relación directa $A$, en el que se denota $a_{i j}$ como el grado en que la estrategia $i$ afecta a la estrategia $j$.

Tabla 2: Matriz inicial de relaciones directas $(A)$ para las estrategias del aspecto financiero

\begin{tabular}{c|ccccc}
\multicolumn{1}{c}{} & E1 & E2 & E3 & E4 & E5 \\
\cline { 2 - 6 } E1 & 0 & 3 & 2 & 1 & 1 \\
E2 & 4 & 0 & 1 & 3 & 1 \\
E3 & 3 & 2 & 0 & 1 & 1 \\
E4 & 1 & 1 & 1 & 0 & 2 \\
E5 & 0 & 3 & 0 & 4 & 0
\end{tabular}

Paso 2: Normalización de la matriz de relaciones directas (Tabla 3). Sobre la base de la matriz de relaciones directas $A$, la matriz normalizada de relaciones directas $M$ se puede obtener a través de fórmulas (1) y (2):

$M=k * A$

$k=\min \left(\left(\frac{1}{\max _{1 \leq i \leq n} \sum_{j=1}^{n}\left|a_{i j}\right|}\right),\left(\frac{1}{\max _{1 \leq j \leq n} \sum_{i=1}^{n}\left|a_{i j}\right|}\right)\right) \quad i, j \in\{1,2,3, \ldots, n\}$

Tabla 3: Matriz normalizada de las relaciones directas para las estrategias del aspecto financiero

\begin{tabular}{cccccc}
\multicolumn{1}{c}{} & E1 & E2 & E3 & E4 & E5 \\
\cline { 2 - 6 } E1 & 0,000 & 0,333 & 0,222 & 0,111 & 0,111 \\
E2 & 0,444 & 0,000 & 0,111 & 0,333 & 0,111 \\
E3 & 0,333 & 0,222 & 0,000 & 0,111 & 0,111 \\
E4 & 0,111 & 0,111 & 0,111 & 0,000 & 0,222 \\
E5 & 0,000 & 0,333 & 0,000 & 0,444 & 0,000
\end{tabular}

Paso 3: Obtención de la matriz de relación total. Una vez que se ha obtenido la matriz de relación directa $M$ normalizada, la matriz $S$ de relación total se puede obtener mediante la fórmula (3), donde I es la matriz de identidad.

$$
\begin{aligned}
& S=M+M^{2}+M^{3}+\cdots=\sum_{i=1}^{\infty} M^{i} \\
& S=M(I-M)^{-1}
\end{aligned}
$$

Paso 4: Determinar el grupo despachador y el grupo receptor. Se utilizan aquí los valores de $D-R$ y $D+R$, donde $R$ es la suma de las columnas y $D$ es la suma de filas en la matriz $S$, según las fórmulas (4) - (6). El criterio que se aplica es que aquellos valores positivos de $D-R$ tienen una mayor influencia sobre otros y se asume que tiene una prioridad más alta y se le llama Causa; mientras que los otros que tienen valores negativos de $D-R$ reciben más influencia de otros y se supone que tienen una prioridad más baja y se llama Receptor. Por otro lado, el valor de $D+R$ indica el grado de relación entre cada estrategia con otras y las estrategias que tienen mayor valores de $D+R$ tienen más relación con otras y las que tienen valores pequeños de $D+R$ tienen menos relación con las demás. 


$$
\begin{aligned}
S & =\left\lfloor s_{i j}\right\rfloor_{n \times n} \quad i, j \in\{1,2,3, \ldots, n\} \\
D & =\sum_{j=1}^{n} s_{i j} \\
R & =\sum_{i=1}^{n} s_{i j}
\end{aligned}
$$

\begin{tabular}{|c|c|c|c|c|c|c|c|c|c|}
\hline & E1 & E2 & E3 & E4 & E5 & D & $D+R$ & $D-R$ & \\
\hline E1 & 0,720 & 0,977 & 0,582 & 0,825 & 0,548 & 3,652 & 7,642 & $-0,338$ & Receptor \\
\hline E2 & 1,127 & 0,835 & 0,575 & 1,082 & 0,633 & 4,253 & 8,454 & 0,051 & Causa \\
\hline E3 & 0,956 & 0,899 & 0,401 & 0,802 & 0,540 & 3,597 & 5,871 & 1,324 & Causa \\
\hline E4 & 0,562 & 0,608 & 0,363 & 0,532 & 0,511 & 2,576 & 6,859 & $-1,707$ & Receptor \\
\hline E5 & 0,625 & 0,882 & 0,353 & 1,042 & 0,438 & 3,340 & 6,010 & 0,670 & Causa \\
\hline $\mathrm{R}$ & 3,990 & 4,201 & 2,274 & 4,283 & 2,670 & & & & \\
\hline
\end{tabular}

Tabla 4: Matriz de relaciones totales para las estrategias propuestas para el aspecto financiero

Paso 5: Establecer el valor del umbral y obtener el diagrama de impacto. El diagrama de impacto también conocido como diagrama causal se puede obtener mediante el mapeo del conjunto de datos de $(D+I, D$ $R$ ), donde el eje horizontal $D+I$ y el eje vertical $D-R$, proporcionan información valiosa para la toma de decisiones. Para obtener un diagrama adecuado, el tomador de decisiones debe establecer un valor umbral para el nivel de influencia. Sólo algunos aspectos, cuya influencia nivel en la matriz $S$ es superior al valor umbral, se pueden elegir y traspasar al diagrama de impacto. Si el valor de umbral es demasiado bajo, el mapa será demasiado complejo para mostrar la información necesaria para la toma de decisiones. Si el valor umbral es demasiado alto, muchos aspectos se presentan como aspectos independientes sin mostrar las relaciones con los otros aspectos. Este valor puede ser referido al promedio de los valores contenidos en la matriz S (Wu et al., 2010).

Paso 6: Obtención del mapa de relaciones de influencia (MRI). Para visualizar las relaciones complejas causales entre las estrategias se utiliza un modelo estructural visible, que puede mostrar el MRI a partir de los valores de $D+I$ y $D-I$, representados en el eje x y el eje y, respectivamente (Wang et al., 2012). En la Fig. 7 se presenta el MRI de las estrategias para el aspecto analizado y en la tabla 5 muestra la matriz de influencias neta de las estrategias en el caso de este proyecto, valores que se obtienen aplicando la ecuación (7). Usando este mapa la administración puede visualizar la diferencia entre las estrategias despachadoras y las estrategias receptoras. Además, la siguiente red de influencia de la matriz $\mathrm{N}$ puede ser utilizada para evaluar la intensidad del efecto de una estrategia sobre otra:

$$
N=s_{i j}-s_{j i}
$$

Tabla 5: Matriz de influencias neta de las estrategias para el aspecto financiero.

\begin{tabular}{lccccc}
\multicolumn{1}{c}{} & E1 & E2 & E3 & E4 & E5 \\
\cline { 2 - 6 } E1 & & & & & \\
E2 & 0,150 & & & & \\
E3 & 0,373 & 0,324 & & & \\
E4 & $-0,263$ & $-0,474$ & $-0,439$ & & \\
E5 & 0,078 & 0,249 & $-0,187$ & 0,531
\end{tabular}




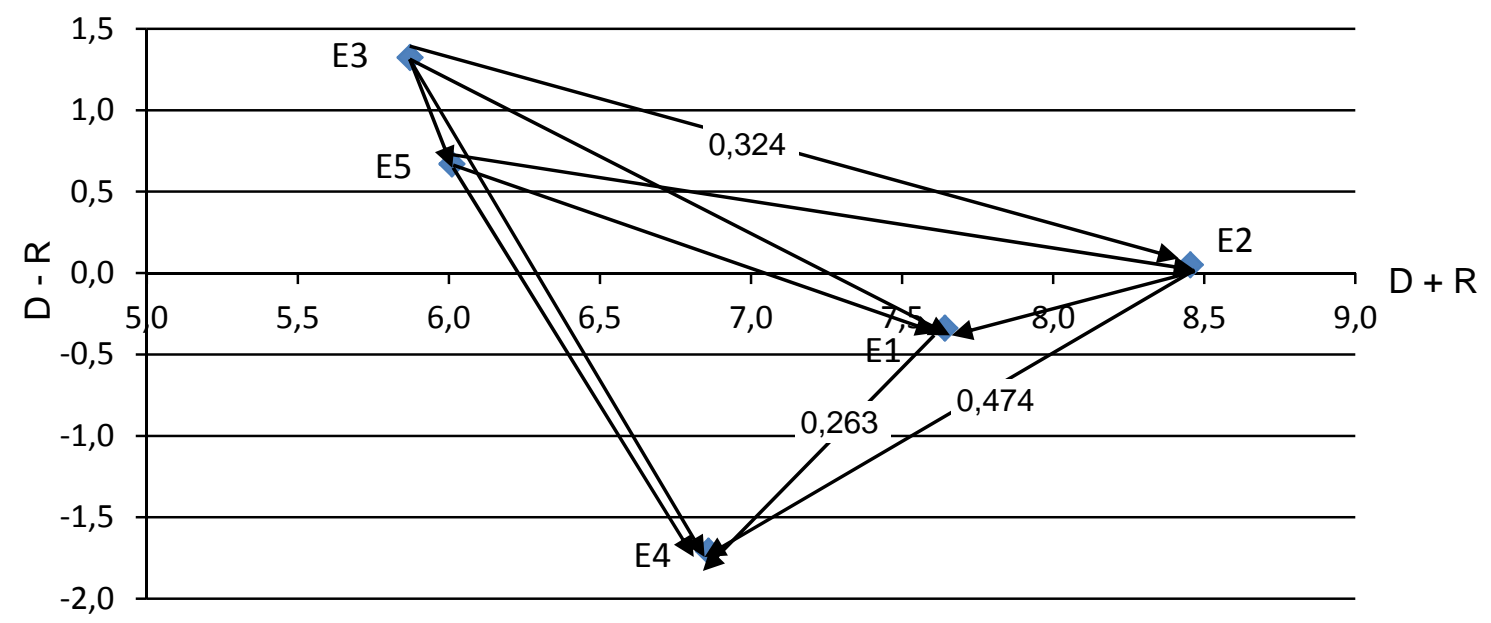

Fig. 7: MRI para las estrategias del aspecto financiero

La Tabla 4 muestra los valores de $D i+R i$ y $D i-R i$ para cada estrategia. Los resultados muestran que los valores de $D i$ - Ri son positivos para las estrategias E2, E3 y E5, que por lo tanto pertenecen al grupo de causas. En general, si la administración desea mejorar la gestión de las estrategias que pertenecen al grupo receptor, no debe descuidar el control de las estrategias del grupo de causas ya que ellas tienen una fuerte interrelación con el resto y un bajo rendimiento puede afectar negativamente sobre el resto.

En la Fig. 7 se presenta el MRI de las estrategias a primer nivel del proyecto de mejoramiento. Usando este mapa, los responsables del proyecto pueden visualizar la diferencia entre las estrategias causa (E2, E3 y E5) y las estrategias afectadas (E1 y E4). Además, la red de influencia de la matriz puede ser utilizada para evaluar la intensidad del efecto de una estrategia sobre otra. La tabla 5 muestra la matriz de influencia neta de las estrategias en el caso del proyecto. Por ejemplo, la influencia neta sobre E4 es bastante mayor desde la estrategia E2 que desde E1 lo que confirma el hecho de provenir desde el grupo causa, y esta información permite a los administradores jerarquizar las estrategias y así asignar recursos humanos y financieros mejor direccionados.

\section{CONCLUSIONES}

La aplicación de esta metodología no termina con el enunciado de las estrategias, sino que es comienzo de una etapa de largo alcance, que deberá ahora ser llevada a cabo por las personas que se desempeñan en la gestión del mantenimiento. Así, a modo de ejemplo, el enunciado de las estrategia E2 dice: "Elaborar un presupuesto más preciso vía hacer un análisis de indicadores de eficiencia en la mantenibilidad" con sus actividades relacionadas de medir la diferencia del gasto estimado y del real por período versus número de indicadores analizados y la eliminación de sus causas raíz de los desvíos, tiene por detrás un conjunto de sub-actividades cuya ejecución si es importante, como ser: actualización continua con datos reales de los gastos directos e indirectos del sistema de información, evaluación del sistema de información acerca de la entrega completa de todos los datos necesarios, emisión de ordenes de trabajo con datos referenciales y recopilación de los datos allí recogidos, capacitación en herramientas de evaluación para su análisis efectivo, definición de actividades para eliminar las causas raíces de los desvíos, control de la aplicación de esas actividades, entre otras. Lo anterior lleva a reforzar el hecho de que hay que disponer de una metodología eficiente que enfoque los esfuerzos del grupo de mantenimiento hacia aquellas actividades que les reportará una mayor eficiencia en su gestión.

Lo que se mostró en este trabajo es una parte de toda la aplicación de la metodología para demostrar la eficacia de esta. Quedó en manos de la administración del mantenimiento de las empresas, que facilitaron la aplicación de esta, continuar con el desarrollo de la aplicación y mantener o aumentar la cultura de evaluación entre sus integrantes, pero, lo que es cierto es que la aplicación y desarrollo de esta metodología fue una gran oportunidad que tuvieron para enumerar sus falencias y proponer las alternativas de solución aplicables a su situación, hecho que se logró a cabalidad tal como lo demuestra la aplicación en su totalidad.

Queda como trabajo a futuro elaborar un procedimiento basado en MCDA para formalizar el sistema de información necesario para apoyar el desarrollo de las estrategias y análisis de los desvíos de estas. 


\section{REFERENCIAS}

Andriani P. y Hall R., Managing knowledge associated with innovation. Journal of Business Research 56, pp. 145- 152, (2003).

Bana e Costa C. A., Carvalho Oliveira R., Assigning priorities for maintenance, repair and refurbishment in managing a municipal housing stock. European Journal of Operational Research 138, pp. 380-391, (2002).

Bana e Costa C.,Carnero M.,Oliveira M., A multi-criteria model for auditing a Predictive Maintenance Programme. European Journal of Operational Research 217, pp. 381-393, (2012)

Bana e Costa C.,Oliveira R.,Assigning priorities for maintenance, repair and refurbishment in managing a municipal housing stock. European Journal of Operational Research 138, pp.380-391, (2002).

Bhuiyan N., Baghel A., An overview of continuous improvement: from the past to the present. Management Decision Vol. 43 No. 5, pp. 761-771, (2005).

Bing Xu B., Ouenniche J., Performance evaluation of competing forecasting models: A multidimensional framework based on MCDA Expert Systems with Applications 39, No 9, pp. 8312-8324, (2012).

Büyükdamgaci G., Process of organizational problem definition: how to evaluate and how to improve. Omega 31, pp. 327 - 338, (2003).

Büyüközkan G., Öztürkcan D., An integrated analytic approach for Six Sigma project selection. Expert Systems with Applications 37, pp. 5835-5847, (2010)

Chaharbaghi K., Willis R., Strategy: the missing link between continuous revolution and constant evolution. International Journal of Operations \& Production Management, Vol 18, No. 9/10. pp. 1017-1027, (1998).

Crespo A., y otros autores., The maintenance management framework A practical view to maintenance management. Journal of Quality in Maintenance Engineering, Vol. 15 No. 2, pp. 167-178, (2009).

Ensslin S. R., y otros autores, Uma Metodologia Multicritério (Mcda-C) Para Apoiar O Gerenciamento Do Capital. Ram - Revista de Administração Mackenzie Volume 9, N. 7, pp. 136-163, (2008).

Ensslin, L., Montibeller Neto, G., Noronha, S. M., Apoio à decisão: metodologias para estruturação e avaliação multicritério de alternativas. Editora Insular, Florianópolis, (2001).

Espinosa F., Dias A., Salinas G., Análisis de riesgo en el proceso de innovación en la gestión del mantenimiento industrial. Intekhnia Vol. 7, No. 1, pp. 13 - 25, (2012).

Espinosa F., Salinas G. Evaluación de la madurez de la función mantenimiento para implementar innovaciones en su gestión. Información Tecnológica, vol.21, No.3, pp.3 - 12, (2010).

Gervásio H., Simões da Silva L., A probabilistic decision-making approach for the sustainable assessment of infrastructures. Expert Systems with Applications 39, pp. 7121-7131, (2012).

Goncalves A. y otros autores. Multicriteria and multivariate analysis for port performance evaluation. Int. J. Production Economics 140, pp. 450-456, (2012).

Hites R., De Smet Y., Risse N., Salazar M., About the applicability of MCDA to some robustness problems. European Journal of Operational Research 174, pp.322-332, (2006).

Hodgkin J.,Belton V.,Koulouri A., Supporting the intelligent MCDA user: A case study in multi-person multicriteria decision support. European Journal of Operational Research 160, pp. 172-189, (2005).

Hu H., Chiu S., Cheng Ch., Yen T., Applying the IPA and DEMATEL models to improve the order-winner criteria: A case study of Taiwan's network communication equipment manufacturing industry. Expert Systems with Applications 38, No 8, pp. 9674-9683, (2011).

Huang Y., Chang Y., Hsieh S, Sandnes F., An adaptive knowledge evolution strategy for finding nearoptimal solutions of specific problems. Expert Systems with Applications 38, pp.3806-3818, (2011).

Johannessen, J.,Olsen B., Olaisen J., Aspects of innovation theory based on knowledge-management. International Journal of Information Management 19, pp. 121-139, (1999).

Kutucuoglu K.Y., Hamali Z, J., Irani, Sharp J.M., A framework for managing maintenance using performance measurement systems International Journal of Operations \& Production Management, Vol. 21 No. 1/2, pp. 173-194, (2001).

Kwon S., Chun Ch., Kwak R. Relationship between quality of building maintenance management services for indoor environmental quality and occupant satisfaction. Building and Environment 46, No 11, pp. 2179-2185, (2011). 
Lee S., Akin Ö. Shadowing tradespeople: Inefficiency in maintenance fieldwork. Automation in Construction 18, No 5, pp. 536-546, (2009).

Lin Y., Yang Y., Kang J., Yu H., Using DEMATEL method to explore the core competences and causal effect of the IC design service company: An empirical case study. Expert Systems with Applications 38, pp. 62626268, (2011).

Linkov, I., y otros autores., From comparative risk assessment to multi-criteria decision analysis and adaptive management: Recent developments and applications. Environment International 32, pp. 1072-1093, (2006).

Løken E., Use of multicriteria decision analysis methods for energy planning problems. Renewable and Sustainable Energy Reviews, Vol. 11, No 7, pp. 1584-1595, (2007).

Madu C. N., Competing through maintenance strategies. International Journal of Quality \& Reliability Management, Vol. 17 No. 9, pp. 937-948. MCB University Press, 0265-671X, (2000).

Martínez S., Aldeanueva C., y Carrasco J., Modelo de decisión multicriterio para la delección de un operador logístico con el que formar una alianza estratégica. Anales X Congreso de Ingeniería de Organización, Valencia, (2006)

Mavrotas G., Diakoulaki D., Caloghirou Y., Project prioritization under policy restrictions. A combination of MCDA with 0-1 programming. European Journal of Operational Research 171, pp. 296-308, (2006).

Mavrotas G., Trifillis P., Multicriteria decision analysis with minimum information: combining DEA with MAVT. Computers \& Operations Research 33, pp. 2083-2098, (2006)

Nickerson, J. A., Zenger, T. R., A Knowledge-Based Theory of the Firm-The Problem-Solving Perspective. Organization Science Vol. 15, No. 6, pp. 617-632, (2004).

Nollet J., Ponce S., Campbell M., About "strategy" and "strategies" in supply management. Journal of Purchasing \& Supply Management 11, pp. 129-140, (2005).

Okeola O.G.,. Sule B.F., Evaluation of management alternatives for urban water supply system using Multicriteria Decision Analysis. Journal of King Saud University - Engineering Sciences 24, No 1, pp. 19-24, (2012).

Okumus F., Towards a strategy implementation framework. International Journal of Contemporary Hospitality Management, 13/7, pp. 327-338, (2001).

Padhy, R.K., Sahu, S., A Real Option based Six Sigma project evaluation and selection model, International Journal of Project Management, Volume 29, Issue 8, pp. 1091-1102, (2011).

Petri A., Heini I., Implementing strategies successfully. Integrated Manufacturing Systems, 13/6, pp. 415418, (2002).

Roux, O., Duvivier, D., Dhaevers, V., Meskens, N., Artiba, A. Multicriteria approach to rank scheduling strategies. International Journal of Production Economics 112, pp. 192-201, (2008).

Sanders D., Smidt R., Statistic: a first course, sixth edition. Ed. Mc Graw Hill Higher Education, (2000).

Sanjay K. Singh, Hugh J. Watson, Richard T. Watson., EIS support for the strategic management process. Decision Support Systems, 33 pp. 71- 85, (2002).

Tsang A., Strategic dimensions of maintenance management Journal of Quality in Maintenance Engineering, Vol. 8 No. 1, pp.7-39, (2002).

Uriona M., Varvakis G., Balanced Scorecard Dinámico en organizaciones intensivas en conocimiento: el caso de una empresa brasilera de desarrollo de software. Revista de dinámica de sistemas Vol. 4 Núm. 1, (Julio 2008).

Wang W-C., Lin Y-H., Lin C-L., Chung C-H., Lee M-T., DEMATEL-based model to improve the performance in a matrix organization. Expert Systems with Applications 39, pp. 4978-4986, (2012).

Winkler R. L., Clemen, R. T., Multiple experts vs. multiple methods: combining correlation. Assessments Decision Analysis Vol. 1, No. 3, pp. 167-176, (2004).

Wu H-H., Shieh J-I., Li Y., Chen H-K., A Combination of AHP and DEMATEL in evaluating the criteria of employment service outreach program personnel. Information Technology Journal 9 (3), pp.569-575, (2010).

Wu W-W., Choosing knowledge management strategies by using a combined ANP and DEMATEL approach. Expert Systems with Applications 35, pp. 828-835, (2008). 
